$\begin{array}{cc}\text { ACADEMIA ROMÂNĂ } & \text { Rev. Roum. Chim., } \\ \text { 2021.66(2), 199-204 } \\ \text { Revue Roumaine de Chimie } \\ \text { http://web.icf.ro/rrch/ }\end{array}$

\title{
EFFECT OF SCANNING SPEED ON AFM HEIGHT IMAGES AND 3D SURFACE TEXTURE PARAMETERS EXPLORED ON SMOOTH AND ROUGH POLYMER SURFACES
}

\author{
Iuliana STOICA* and Elena Gabriela HITRUC \\ “Petru Poni” Institute of Macromolecular Chemistry, Iaşi - 700553, Roumania
}

Received October 1, 2020

Atomic force microscopy (AFM) is a powerfull technique developed in the last decades that is widely used for surface morphology investigation at micro and nanometric scale. Along with the environment in which the scan is performed and the type of sample, the scanning conditions play an important role in obtaining the best results in imaging, without any artifacts that may be occure. In the present study, in order to establish the proper scanning conditions, smooth and rough polymer surfaces were used to investigate the influence of the scanning speed on height images and amplitude, hybrid, spatial, and functional 3D surface texture parameters in atomic force microscopy.

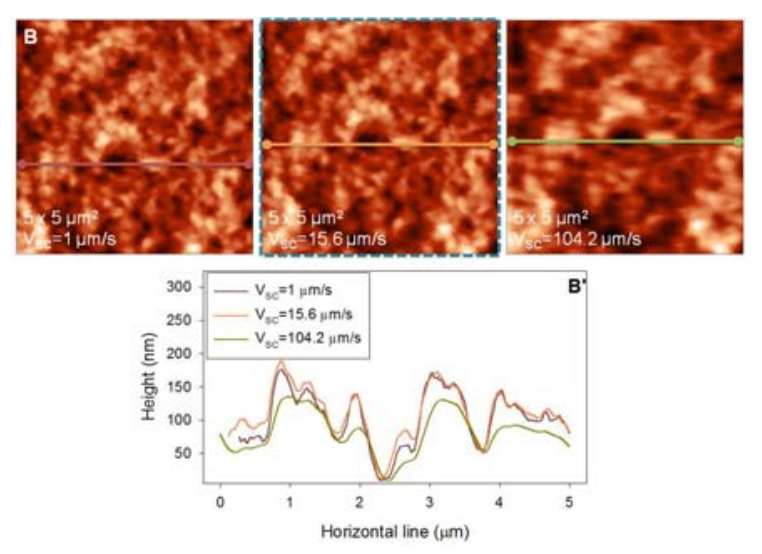

\section{INTRODUCTION}

One of the extremely notable methods involving the surface topography research is the atomic force microscopy (AFM). ${ }^{1}$ This tool is exceptional because of the variety of suitable materials belonging to all research areas. Using this instrument give us the possibility to obtain high resolution surface images and information at the nanoscale which is unavailable to other research techniques. While other microscopy techniques usually involve the term of looking, AFM can be compared to the sense of touching. ${ }^{2}$ The results of scanning are obtained by the instrumentality of a sharp tip which is touching (up, down, right, left) the surface of the sample. ${ }^{3}$ There is a wide variety of materials like ceramics, metals, biological and polymers that atomic force microscopy is able to scan and investigate. The characterization of the surfaces through AFM can be effectuated in air, in vacuum and in liquid environment. ${ }^{4}$ Depending on the investigated sample, there are three different scanning modes available: non-contact, contact and tapping mode. In order to obtain results at a nanoscale resolution, without damaging the surfaces, for polymers the studies are focused on scanning tapping mode of getting images during the tip is intermittently

\footnotetext{
${ }^{*}$ Corresponding authors: stoica_iuliana@icmpp.ro
} 
touching the sample. ${ }^{5}$ During the tapping mode scanning, there are several scanning parameters that must be adjusted depending on the investigated sample, ${ }^{6}$ in order to obtain eloquent measurements, without artifacts. ${ }^{7}$

Previously, our studies were concentrated on the impact of tip geometry, scanning area, pixel size, set-point amplitude ratio and image preprocessing procedure, on height images quality and 3D surface texture parameters, on different polymer films investigations. ${ }^{8}$ Further this work intent to find the optimal values for another important scanning parameter, namely the velocity of scanning, with the purpose of obtaining the best quality of images. The interests regarding this subject start with the development of AFM technique and it keep going in present. T. Sulchek et al. found the fact that increasing the speed of tapping mode AFM scanning brings notable scientific data and practical acquaintance. The mentioned authors demonstrated that the feedback loop is the one which limited the rate (speed) of scanning in AFM tapping mode. A constant value for tapping amplitude is fixed by the speed of feedback loop. Also there were found the limits for optimal scanning speed. ${ }^{9}$ In recent years Sobola \& contributors highlighted that using lower speed of scanning is proper on rough sample while an increased scanning rate is convenient on materials having lower fractal dimension. Thus the specific feature of the samples it is taken into consideration in obtaining high quality AFM measurements. ${ }^{10}$ In this context, we continue our findings regarding the evolution of the amplitude, hybrid, spatial, and functional 3D texture parameters with respect to the scanning speed in AFM imaging. To our knowledge, this type of comparative study on smooth and rough polymer surfaces was not yet presented in profile publications.

\section{RESULTS AND DISCUSSION}

In our days, the investigation of the surface morphology on polymer films is of great importance in revealing the micro and nanoscale topographic features induced by the chemical structure of the polymer, the film formation procedure and other applied treatments. Atomic force microscopy is one of the best techniques for this. In order to obtain correct images, without artifacts, and implicitly right roughness parameters, proper scanning conditions, including scanning speed must be establish, depending on the surface characteristics of the polymers: smooth, with high Young's modulus at room temperature and rough, with low Young's modulus at room temperature. To study the influence of the scan speed on the topographical images and surface texture parameters, AFM imaging was carried out at 33 different scan speeds, starting from $1 \mu \mathrm{m} / \mathrm{s}$ to $104.2 \mu \mathrm{m} / \mathrm{s}$, at least three $5 \times 5 \mu \mathrm{m}^{2}$ images per speed being thus obtained for each smooth surface of poly(BTDA-DDM), prepared using 3,3',4,4'benzophenonetetracarboxylic di-anhydride (BTDA) and 4,4'-diaminodiphenylmethane (DDM) monomers, and rough surface of poly(MDI-PEA-EG), formed by reacting together three chemical constituents: a diisocyanate, namely 4,4'methylenebis(phenyl isocyanate) (MDI), a longchain macrodiol, i.e., poly(ethylene adipate) diol (PEA) and a small molecule chain extender diol such as ethylene glycol (EG). Some representative AFM topographical images recorded at three different scanning speeds (two extreme values, namely $1 \mu \mathrm{m} / \mathrm{s}$ (minimum) and $104.2 \mu \mathrm{m} / \mathrm{s}$ (maximum) and a middle one, $15.6 \mu \mathrm{m} / \mathrm{s}$ ) are presented in Figure 1, along with the corresponding cross-section profiles that follow the same scan line from the height images obtained for poly(BTDA-DDM) (Figure 1A, A') and poly(MDIPEA-EG) (Figure 1B, B').

Usually, in tapping mode, the scan velocity is restricted by the reaction time of the feedback circuit. $^{11,12}$ Therefore, in order to achieve the best possible surface tracking (smallest possible feedback error signal), the feedback gain was set at the value of 0.6. The peak of the resonance curve was found at 97 $\mathrm{kHz}$ for the used cantilever. The quality factor attributed to this probe, calculated as the height of the resonance curve divided by its width at half the maximum height, was 181 . To estimate the response of the cantilever probe, the time constant was computed taking into account the quality factor and the resonance frequency of the probe. ${ }^{13}$ The reduced value of the time constant (3.73) indicates a fast response of the probe, reduced scan error and good image quality, as stated by Orun et al. ${ }^{14}$

According to our experiments performed for both poly(BTDA-DDM) and poly(MDI-PEA-EG) it was found a clear dependence of the height image aspect on the scan speed. At low values of the scan rate, the representative height images from Figure 1 (obtained at 1 and $15.6 \mu \mathrm{m} / \mathrm{s}$ ) and their corresponding profiles reveal clear well-defined morphological features. A possible explanation for the observed phenomena can be that at low scan velocities region the data acquisition and signal processing is faster, providing 
good quality for the AFM images. Still, the best results were obtained around $15.6 \mu \mathrm{m} / \mathrm{s}$. In this case the formations are very pronounced, especially for the rough polymer surface.

Instead, at high scan velocities, mostly after 26 $\mu \mathrm{m} / \mathrm{s}$, the images have poor quality due to the delay of the signal in the feedback loop. As the scanning speed increases, there is an increase in contact between the leading face of the tip and the sides of the polymer surface, leading the probe to be increasingly restrained by the polymer. ${ }^{15}$ The images lose their clarity, the surface formations being not so precisely marked. The representative data presented in Figure 1 for maximum scanning speed of $104.2 \mu \mathrm{m} / \mathrm{s}$ confirmed this affirmation. In the case of smooth polyimide surface, the differences in the height profile are relatively small, of 1-2 $\mathrm{nm}$. One can observe at the side of the image vertical lines running perpendicular to the scan direction. These lines result from the turnaround at the borders of the scan which induce oscillations in the $\mathrm{X}, \mathrm{Y}$ scanner, indicating that the line distance is too long in relation to the assigned time-per-line.

Instead, when the rough polyurethane sample is taken into discussion the green cross-section profile corresponding to $104.2 \mu \mathrm{m} / \mathrm{s}$ reveal important differences in the height profile, even of 50-70 nm. In this way the image obtained after scanning in these conditions it does not even nearly reflect the reality.
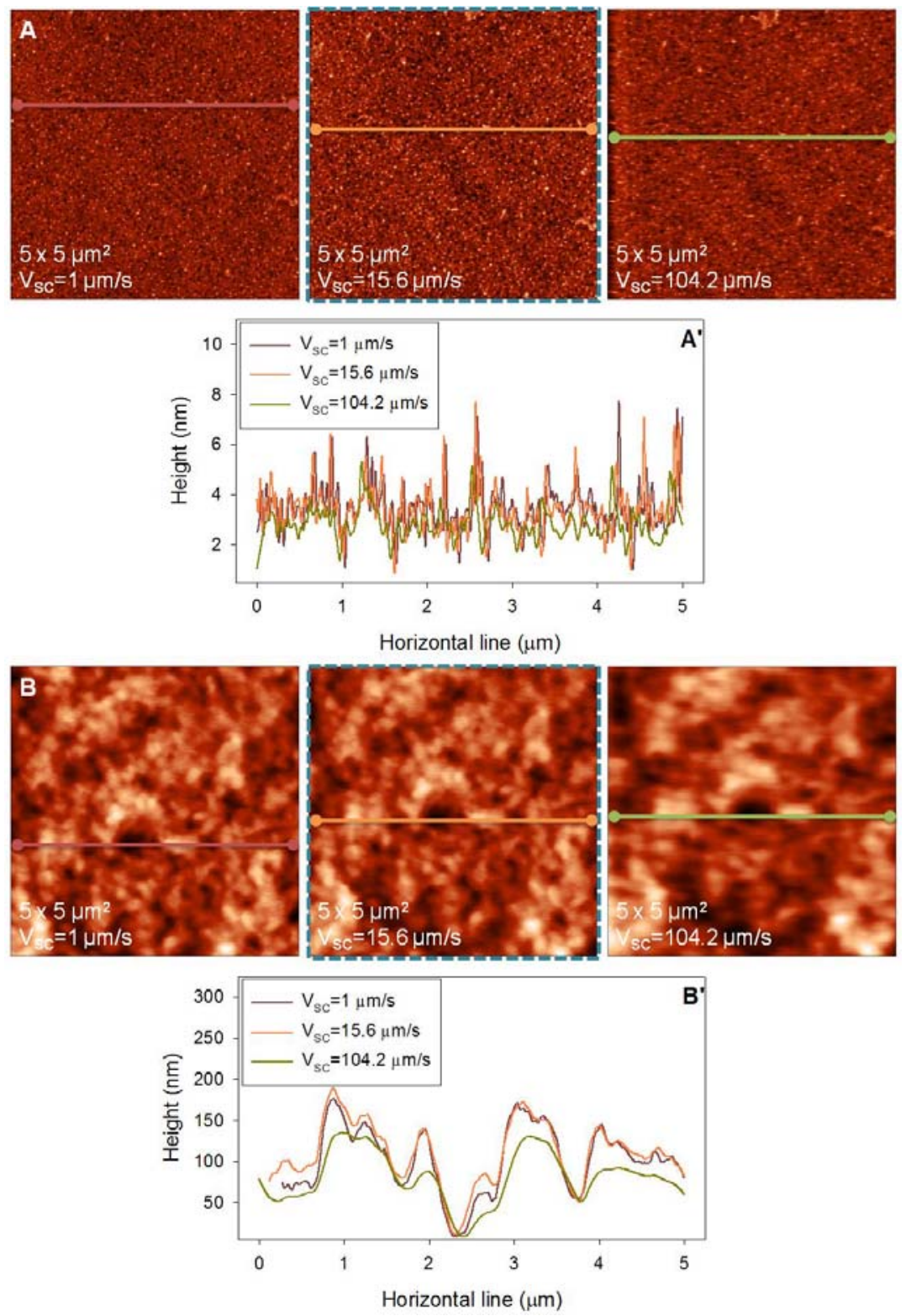

Fig. 1 - Representative height images and corresponding cross-section profiles obtained for poly(BTDA-DDM) (A, A') and poly(MDIPEA-EG) (B, B') samples recorded using different scanning velocities: $1 \mu \mathrm{m} / \mathrm{s}, 15.6 \mu \mathrm{m} / \mathrm{s}$ and $104.2 \mu \mathrm{m} / \mathrm{s}$. The scan area was $5 \times 5 \mu \mathrm{m}^{2}$. 
The fact that the quality of the AFM images depends on the scanning rate, influence also the evolution of the 3D texture parameters, namely of the root mean square roughness $(S q)$, surface area ratio $(S d r)$, surface texture direction index $(S t d i)$, surface bearing index $(S b i)$, core fluid retention index $(S c i)$ and valley fluid retention index $(S v i)$. $S d r$ is used to evaluate the morphology complexity and can be defined as the increment of the total surface area relative to the sampling area. Stdi can be defined as the ratio of the average amplitude sum to the amplitude sum of the dominating direction and shows the degree of surface orientation: if the amplitude sum of all directions is almost similar, the values of the Stdi will be close to 1 and surface will present isotropic morphology; if Stdi values will be close to 0 , the morphology will have a dominant direction (anisotropy). The functional parameters, namely functional indexes (Sbi, Sci, Svi) were evaluated from the Abbott-Firestone curves (surface bearing area ratio curves), based on atomic force microscopy images of the samples surfaces. Sbi is defined as the ratio of the $S q$ of the surface to the height at which the areal material ratio is $5 \%$. Sci is defined as the ratio of the void space volume inside the core region (material ratio between $5 \%$ and $80 \%$ ) per unit area to the $S q$. Svi is defined as the ratio of the void space volume inside valleys region (material ratio between $80 \%$ and $100 \%$ ) per unit area to the $S q$.

$S q, S d r$ and $S t d i$, calculated for the poly(BTDADDM) (Figure $2 \mathrm{~A}, \mathrm{~B}, \mathrm{C}$ ) and poly(MDI-PEA-EG) (Figure 2 D, E, F) samples with respect to the scanning velocity, have a decreasing trend simultaneously with the decrease in quality of the AFM images (see Figure 2). Still, there is a region with a plateau, where their variations are relatively small. Instead, after $26 \mu \mathrm{m} / \mathrm{s}$ these parameters drop abruptly. Also, by increasing the scanning speed, the obtained lower values for $S d r$ suggested the decreasing of the morphology complexity, and for $S t d i$, suggested an incorrect tendency of the surface texture to become orientated on the scanning direction, in other words to present anisotropy. This phenomenon occurs faster when the surface of the sample is smooth, as may be seen in Figure $2 \mathrm{C}$, probably due to the polyimide high Young's modulus.
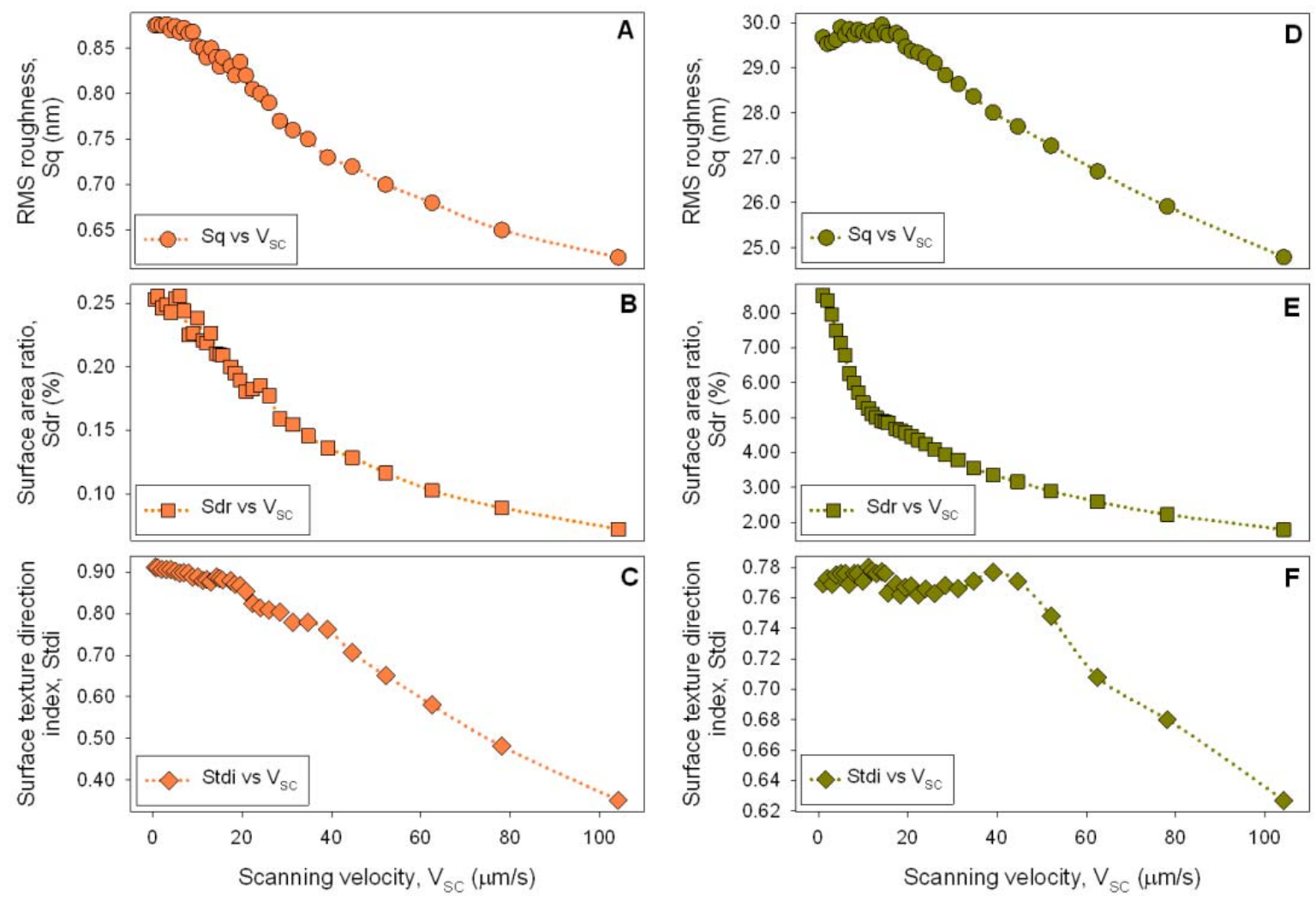

Fig. 2 - Root mean square roughness, surface area ratio and surface texture direction index calculated for the poly(BTDA-DDM) (A, B, C) and poly(MDI-PEA-EG) (D, E, F) samples with respect to the scanning velocity. 

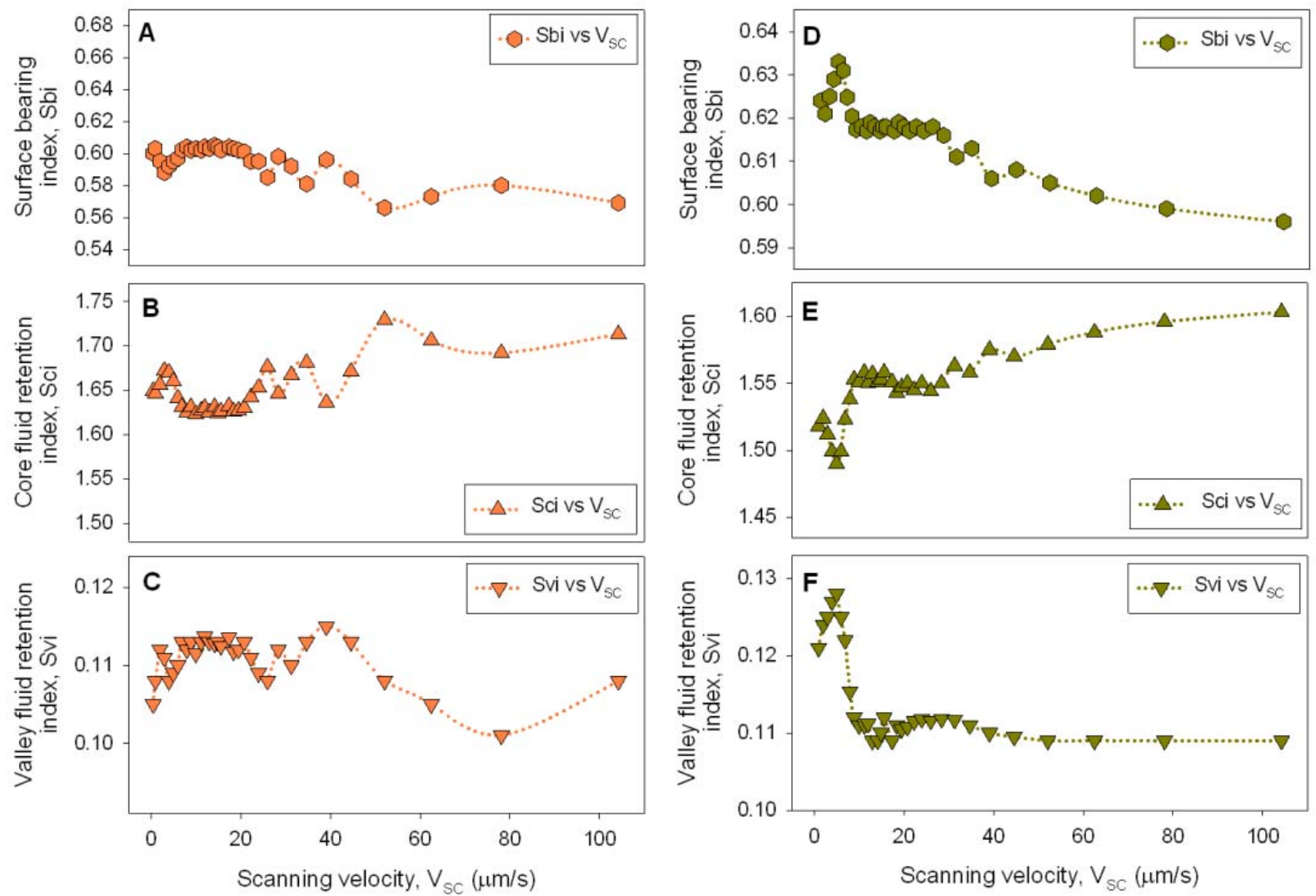

Fig. 3 - Surface bearing index, core fluid retention index and valley fluid retention index calculated for the poly(BTDA-DDM) (A, $\mathrm{B}, \mathrm{C})$ and poly(MDI-PEA-EG) (D, E, F) samples with respect to the scanning velocity.

The evolution of the AFM functional parameters with scanning speed calculated for both samples is shown in Figure 3. It can be easily noticed that surface bearing index (Sbi), core fluid retention index $(S c i)$ and valley fluid retention index $(S v i)$ which depict both sample shape and function, remain almost constant on different domains of scanning velocity, namely for poly(BTDA-DDM) sample between $6.9 \mu \mathrm{m} / \mathrm{s}$ and $20.8 \mu \mathrm{m} / \mathrm{s}$, and for poly(MDI-PEA-EG) sample between $8.9 \mu \mathrm{m} / \mathrm{s}$ and $28.4 \mu \mathrm{m} / \mathrm{s}$. Due to the morphological apparent modifications, at high scan velocities, the bearing properties are tainted.

\section{EXPERIMENTAL}

\section{Polymer films}

The polyimide film poly(BTDA-DDM) of about $60 \mu \mathrm{m}$ in thickness was obtained by thermal imidization. Details regarding the synthesis of this polymer were presented somewhere else. ${ }^{16,17}$ The Young's modulus of the polyimide at room temperature was $3 \mathrm{GPa}$. The elastomeric polyurethane film poly(MDI-PEA-EG) with thickness of $0.3 \mathrm{~mm}$ was obtained by casting the mixture into closed sheet mould for curing at $110^{\circ} \mathrm{C}$ over 24 hours. The synthesis and characterization of this polymer were presented elsewhere. ${ }^{18,19}$ The Young's modulus of the polyurethane at room temperature was $85 \mathrm{MPa}$.

\section{Atomic force microscopy measurements}

The AFM measurement were performed using a Solver PRO-M (NT-MDT Co, Zelenograd, Moscow, Russia) setup, at room temperature, with a commercially available NSG03 (NT-MDT Co, Zelenograd, Moscow, Russia) rectangularshaped silicon cantilever. The cantilever's normal spring constant was determined by Sader's method, ${ }^{20,21}$ using the value of the resonant frequency of $97 \mathrm{kHz}$ and the planar dimensions of the cantilever, namely length $(1=135 \pm 5 \mu \mathrm{m})$, width $(\mathrm{w}=30 \pm 5 \mu \mathrm{m})$ and thickness $(\mathrm{t}=1-2 \mu \mathrm{m})$. The obtained value was $2.4 \mathrm{~N} / \mathrm{m}$. The tip radius was evaluated at $8.6 \pm 0.3$ $\mathrm{nm}$ by means of SPIP ${ }^{\mathrm{TM}}$ software (Scanning Probe Image Processor, Image Metrology, Denmark). The employed method is presented elsewhere ${ }^{8}$. The SEM image obtained for the tip is presented in Figure 4.

For AFM data acquisition and processing, two software developed by the NT-MDT Co were used, namely Nova v.1.26.0.1443 for Solver and Image Analysis P9 3.5.0.2071. The evaluation of the surface roughness and texture was made in terms of root mean square roughness $(S q)$, surface area ratio $(S d r)$, texture direction index $(S t d i)$, surface bearing index $(S b i)$, core fluid retention index $(S c i)$ and valley fluid retention index $(S v i)$, defined elsewhere. ${ }^{22,23}$ 


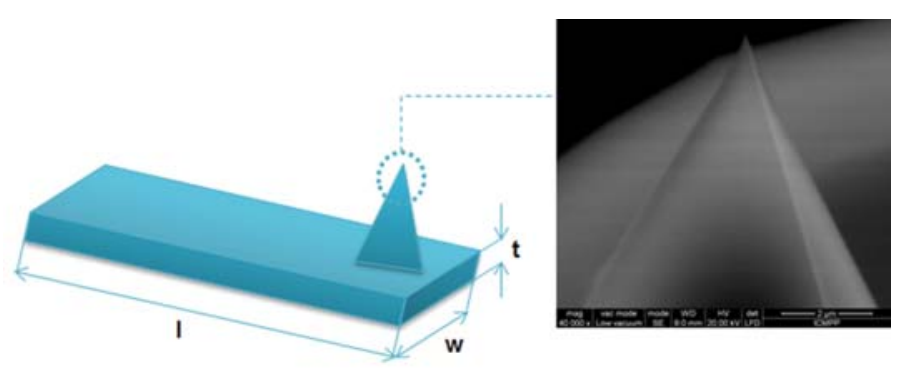

Fig. 4 - Schematic representation of the NSG03 rectangular-shaped silicon cantilever with SEM image of the cantilever's tip

\section{CONCLUSIONS}

In order to establish the proper scanning conditions in atomic force microscopy, in the present study smooth and rough polymer surfaces were used to investigate the influence of the scanning speed on height images and corresponding amplitude, hybrid, spatial, and functional 3D surface texture parameters.

As a result of all the experiments, on may conclude that the AFM images will exhibit good contrast and resolution and the values of 3D texture parameters will remain constant when during intermittent contact mode, a suitable scanning velocity is provided, ranging from $6.9 \mu \mathrm{m} / \mathrm{s}$ to 20.8 $\mu \mathrm{m} / \mathrm{s}$ for smooth sample and from $8.9 \mu \mathrm{m} / \mathrm{s}$ to 28.4 $\mu \mathrm{m} / \mathrm{s}$ for rough sample. Beside these stabile regions, image artifacts will occur, and by default wrong values of the 3D texture parameters that will lead to erroneously interpretations.

Acknowledgements. This work was performed with the support of Roumanian Academy Project "Polymer materials. Correlations of structure, morphology, optical and electrical properties" (P8.3).

\section{REFERENCES}

1. G. Binnig, C.F. Quate and Ch. Gerber, Phys. Rev. Lett., 1986, 56, 930-933.

2. A. Pfau, A. Janke and W. Heckmann, Surf. Interf. Anal., 1999, 27, 410-417.

3. S.M. Flores, J.L. Toca-Herrera, Nanoscale, 2009, 1, 4049.
4. D. Passeri, M. Rossi, E. Tamburri and M. L. Terranova, Anal. Bioanal. Chem., 2013, 405, 1463-1478.

5. L. C. Sawyer, D. T. Grubb and G. F. Meyers, "Polymer Microscopy", Springer, New York, 2008.

6. R. García and A. San Paulo, Phys Rev. B, 1999, 60, 4961-4967.

7. A. Kühle, A. H. Sørensen, J. B. Zandbergen and J. Bohr, Appl. Phys. A, 1998, 66, S329-S332.

8. I. Stoica, E .G. Hitruc, D. Timpu, V. Barboiu and D. S. Vasilescu, SCANNING, 2015, 37, 335-349.

9. T. Sulchek, G. G. Yaralioglu, C. F. Quate and E. L. Ginzton, Rev. Sci. Instrum., 2002, 73, 2928.

10. D. Sobola, Ş. Țalu, S. Solaymani and L. Grmela, Microsc Res Tech., 2017, 80, 1328-1336.

11. T. Sulchek, G. G. Yaralioglu, C. F. Quate and E. L. Ginzton, Rev. Sci. Instrum., 2002, 73, 2928.

12. S.-M. Lin and C.-C. Lin, Precision Engineering, 2009, 33, 71-377.

13. T. R. Albrecht, P. Grtitter, D. Horne and D. Rugar, J. Appl. Phys., 1991, 69, 668-673.

14. B. Orun, S. Necipoglu, C. Basdogan and L. Guvenc, Rev. Sci. Instrum., 2009, 80, 063701(1-22).

15. G. S. Watson, C. L. Brown, S. Myhra, N. C. Roch, S. Hu and J. A. Watson, Proc. SPIE 6037, Device and Process Technologies for Microelectronics, MEMS, and Photonics IV, 2005, 60371B.

16. M. Cristea, D. Ionita, C. Hulubei, D. Timpu and B. C. Simionescu, Polymer, 2011, 52, 1820-1828.

17. D. Popovici, C. Hulubei, V. Cozan, G. Lisa and M. Bruma, High Perform. Polym., 2012, 24, 194-199.

18. C. Prisacariu, C. P. Buckley and A. A. Caraculacu, Polymer, 2005, 46, 3884-3894.

19. C.P. Buckley, C. Prisacariu and C. Martin, Polymer, 2010, 51, 3213-3224.

20. J. E. Sader, J. W. M. Chon and P. Mulvaney, Rev. Sci. Instrum., 1999, 70, 3967-3969.

21. J. E. Sader, J. Pacifico, C. P. Green and P. Mulvaney, J. Appl. Phys., 2005, 97, 124903(1-7).

22. ASME B46.1, 2009.

23. ISO 25178-2, 2012. 